\title{
Surface Differential Rotation of Evolved Fast Rotators
}

\author{
P. Petit \\ Observatoire Midi-Pyrénées, 14 avenue Edouard Belin, 31400 Toulouse, \\ France \\ Centro de Astrofisica da Universidade do Porto, rua das Estrelas, \\ 4150-762 Porto, Portugal (petit@astro.up.pt)
}

\section{J.-F. Donati}

Observatoire Midi-Pyrénées, 14 avenue Edouard Belin, 31400 Toulouse, France (donati@ast.obs-mip.fr)

The MuSiCoS collaboration

G.A. Wade (Royal Military College of Canada), J.D. Landstreet, T.A.A. Sigut, S.L.S. Shorlin (University of Western Ontario, Canada), S. Bagnulo (European Southern Observatory, Chile), T. Lüftinger (Institut für Astronomie, Vienna), S. Strasser (University of Minnesota, USA), J.M. Oliveira (Keele University, UK), M. Aurière, F. Lignières, D. Mouillet (Observatoire Midi-Pyrénées, France), F. Paletou (Observatoire de la Côte d'Azur, France)

\begin{abstract}
Zeeman-Doppler Imaging enables one to estimate the short term temporal evolution of surface brightness and magnetic structures, under the effect of differential rotation. We present here spectropolarimetric observations secured between 1998 and 2002 for two evolved active stars: the K1 subgiant of the RS CVn system HR 1099 and the single FK Com giant HD 199178. Differential rotation is detected both from brightness and magnetic images, indicating that the rotational shear, roughly solar in magnitude for the single star, is significantly weaker in the binary system. This result suggests that tidal forces, rather than stellar evolution, could be responsible for the lower rotational shear and thus the longer spot lifetime reported for binary systems.
\end{abstract}

\section{Differential Rotation and Magnetic Activity}

Differential rotation is one of the basic ingredients invoked to explain the generation of the solar magnetic field, through its ability to transform a large-scale poloidal magnetic field into a stronger toroidal component. This so-called " $\Omega$ effect" is believed to take place in the thin layer separating the radiative core from the convective envelope of the Sun: the tachocline. Despite this interest, much details of this general principle are still poorly understood. A promising option to progress on this issue is the systematic investigation of differential rotation on a sample of active stars other than the Sun, in order to estimate the impact on the rotation law of various stellar parameters (such as the rotation period, 
the stellar mass, the depth of the convective zone, the evolutionary stage, or the tidal forces operating on late-type components of close binaries). Another potentially fruitfull investigation concerns the feedback of the magnetic field on the differential rotation, which may produce temporal fluctuations of the rotational shear (Applegate 1992). This effect is subtle on the Sun (Vorontsov et al. 2002) but is likely to reach a higher level on extremely active objects like fast rotating stars.

Zeeman-Doppler Imaging (hereafter ZDI, Donati \& Brown 1997) gives access not only to the photospheric distribution of magnetic regions and cool spots on fast rotators, but also to the temporal evolution of these structures under the effect of surface differential rotation. We first recall the technics developed to conduct surface differential rotation analysis in the framework of ZDI, as well as the first results derived for a sample of young active stars. We then turn towards two evolved fast rotators : the K1 subgiant of the RS CVn system HR 1099 and the FK Com giant HD 199178, using both cool spots and magnetic photospheric structures as tracers of their large-scale surface flows. We finally suggest some physical processes, as well as further observational tests, to interpret the observed surface shears.

\section{Detection of Surface Differential Rotation : Method and First Results}

\subsection{Estimating Surface Differential Rotation from Direct Data Fit- ting.}

Classical analysis of surface differential rotation involving ZDI are performed by the comparison of successive images of the same object (recorded a few days apart), in order to detect the relative motion of active regions under the effect of the rotational shear. Such a simple method, successful in the case of very rapid rotators (with rotation period smaller than, or close to, one day), may suffer from important biases for objects with rotation periods of the order of a few days (Petit et al. 2002), thus preventing the use of this kind of strategy for close binaries (like RS CVn systems) or FK Com stars.

To circumvent this drawback, we rather consider the whole series of spectra secured at one epoch and reconstruct one single image of the stellar photosphere, assuming into the inversion process itself that the surface undergoes a differential rotation law of the form :

$$
\Omega(l)=\Omega_{\mathrm{eq}}-d \Omega \sin ^{2} l
$$

where $\Omega(l)$ is the rotation rate at latitude $l, \Omega_{\text {eq }}$ the rotation rate of the equator and $d \Omega$ the difference in rotation rate between the pole and the equator. The parameter pair is determined as being the one providing the best fit to the data (at a constant information content of the reconstructed image). This method was tested through numerical simulations (Petit et al. 2002) and turns out to behave very well for synthetic data sets similar in quality to that used in the present study. 


\subsection{First Results for Pre-Main Sequence Fast Rotators}

Several detections of differential rotation have already been performed on a sample of young active fast rotators of spectral types $\mathrm{G}$ and $\mathrm{K}$, using either a classical method based on image comparison (Donati et al. 2000, Barnes et al. 2000 ) or a direct data fitting (Donati et al. 2003b). For all stars presenting a surface shear, the equator is rotating faster than the pole, with laptimes (time for the equator to lap the pole by one complete rotation cycle) roughly solar in magnitude ( $40 \mathrm{~d}$ to $110 \mathrm{~d}$, while the laptime of the Sun is roughly equal to $120 \mathrm{~d}$ ). This first result demonstrates that the laptime is mostly independent of the rotation rate (since these objects rotate 20 to 80 times faster than the Sun). Another finding is that the shear intensity tends to increase with the stellar mass (Donati et al. 2000). Donati et al. (2003b) also report definite temporal fluctuations of the surface shear on several objects, and note that the shear of magnetic regions is systematically stronger than that of cool spots.

In the next sections, we investigate how these conclusions hold for more evolved objects (presenting a much deeper convective envelope than the young stars observed so far) and how tidal forces may impact on the surface differential rotation in the case of an object belonging to a close binary system.

\begin{tabular}{|c|cc|cc|}
\hline \multirow{2}{*}{ Object } & \multicolumn{2}{|c|}{ Cool spots } & \multicolumn{2}{c|}{ Magnetic regions } \\
& $\begin{array}{c}\Omega_{\mathrm{eq}} \\
\mathrm{rad} \mathrm{d}^{-1}\end{array}$ & $\begin{array}{c}d \Omega \\
\mathrm{mrad} \mathrm{d}^{-1}\end{array}$ & $\mathrm{rad} \mathrm{d}^{-1}$ & $\begin{array}{c}d \Omega \\
\mathrm{mrad} \mathrm{d}^{-1}\end{array}$ \\
\hline HR 1099 & $2.2241 \pm 0.0004$ & $15.2 \pm 0.8$ & $2.2232 \pm 0.0009$ & $9.7 \pm 1.8$ \\
HD 199178 & - & - & $1.93 \pm 0.02$ & $70 \pm 30$ \\
& & & & \\
\hline
\end{tabular}

Table 1. Surface rotation parameters derived for HR 1099 and HD 199178. $\Omega_{\mathrm{eq}}$ and $d \Omega$ are listed from surface brightness inhomogeneities (columns 2 and

3 ) and from magnetic tracers (columns 4 and 5).

\section{Application to Evolved Fast Rotators}

\subsection{Observations}

The data sets we used for this study were secured with the MuSiCoS spectropolarimeter at the Télescope Bernard Lyot and with UCLES (fibre-fed by the polarimeter SemelPol) at the Anglo-Australian Telescope. Several observing campaigns were conducted for HR 1099 with both instruments from January 1998 to January 2002, while HD 199178 was observed with MuSiCoS from December 1998 to July 2002. Each data set covers a timespan consistent with the lifetime of the photospheric features used to track the surface flows, i.e. typically 4 weeks and 2 weeks for HR 1099 and HD 199178 respectively. We thus obtain 4 (resp. 6) data sets, constituting a total data base of 420 unpolarised and 104 circularly polarised spectra (resp. 208 and 51 ).

The data reduction was performed with ESpRIT (Donati et al. 1997). Least-Square Deconvolution (Donati et al. 1997) was employed to simultaneously extract the information from most spectral features of the echellograms, so 


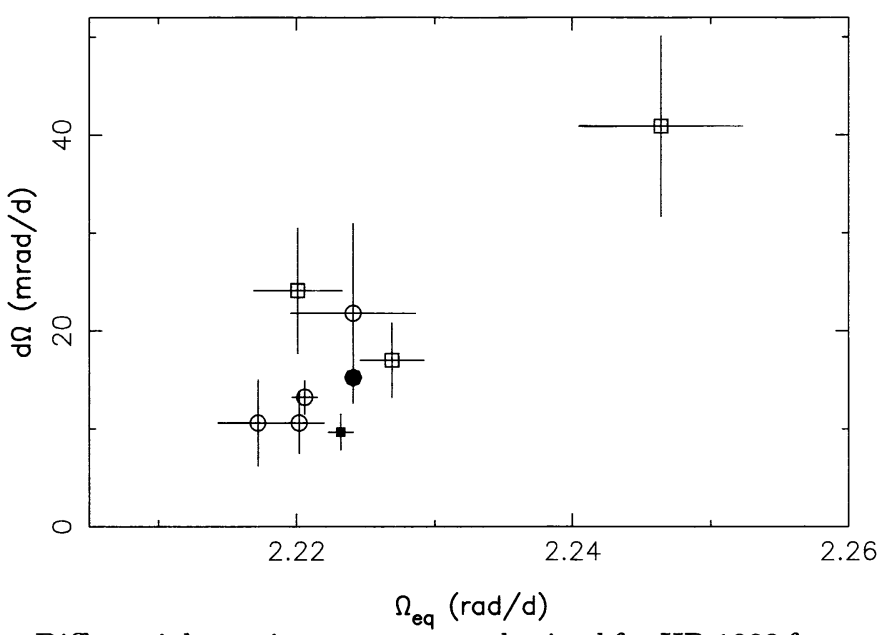

Figure 1. Differential rotation parameters obtained for HR 1099 from several observing epochs. The circles (resp. squares) show estimates derived from magnetic (resp. brightness) tracers. The black-filled symbols represent the parameters obtained from bi-site observations using the TBL and the AAT (corresponding to the values reported in Table 1).

that multiplex gains of the order of 40 were usually achieved, producing circularly polarised mean profiles with a $\mathrm{S} / \mathrm{N}$ reaching as much as 20,000 per $2 \mathrm{~km} . \mathrm{s}^{-1}$ velocity bin for the best data sets of HR $1099\left(7,000\right.$ per $4 \mathrm{~km} \cdot \mathrm{s}^{-1}$ velocity bin for HD 199178).

\subsection{The K1 Subgiant of the RS CVn System HR 1099}

The K1 primary subgiant of the RS CVn system HR 1099 is one of the most active stars in the whole sky. A strong tidal coupling forces both late-type components of the system to corotate with an orbital period equal to $2.83 \mathrm{~d}$. The brightness and magnetic topologies of the primary component has been mapped in several papers (see e.g. Donati et al. 2003a), revealing a complex, varying photospheric magnetic field.

Both brightness inhomogeneities and magnetic regions can be used to track the photospheric differential rotation on this star (Petit et al. 2003a). The estimates obtained for different observing epochs are represented in Fig. 1. Differential rotation is repeatedly detected, to within as much as $18 \sigma$ for our best data set (Table 1). As in the solar case, the equator is rotating faster than the pole, but the intensity of the differential rotation is about 4 times weaker than on the Sun, with a laptime of the order of $480 \mathrm{~d}$.

We do not detect any systematic difference of level between the rotational shears of cool spots and magnetic regions, but we report a maginal time-variability of the differential rotation (see the upper-right point of Fig. 1, corresponding to February 1998 observations). However, the error bars are usually too large to conclude on the analysis of such subtle effects, and the much smaller uncertainties provided by bi-site observations (black-filled dots, Fig. 1) suggest that systematic multi-site campaigns are necessary for this kind of investigation. 


\subsection{The FK Com Giant HD 199178}

A photospheric magnetic field was recently detected and mapped for the FK Com G5 giant HD 199178 (Petit et al. 2003b). This star is more massive than HR $1099\left(1.65 \mathrm{M}_{\odot}\right.$ vs. $\left.1 \mathrm{M}_{\odot}\right)$ and is a single star, but this is also an object arriving on the giant branch. Furthermore, its rotation period is close to that of HR 1099 ( $3.3 \mathrm{~d})$ and the photospheric magnetic fields of both objects share several general characteristics, with a predominance of a nearly axi-symmetric azimuthal component, structured as rings of opposite polarities encircling the pole at different latitudes.

Differential rotation is particularly difficult to measure on this star for two main reasons. First, the $\mathrm{S} / \mathrm{N}$ of the corresponding data sets is usually below the values one can reach with HR 1099. Moreover, the lifetime of photospheric tracers is significantly shorter on this target, thus forcing to reduce the timespan of data sets, and therefore limiting the longitude shifts of the tracers during data collection. Owing to these constraints, no detection was achieved from the intensity profiles, and only two definite detections (to within $2 \sigma$ ) were obtained from magnetic structures (see Table 1). Once again, the equator is rotating faster than the pole but the surface shear is significantly stronger than on HR 1099, with a laptime roughly equal to $90 \mathrm{~d}$.

\section{Discussion}

This work reports an estimate of the surface differential rotation on two evolved fast rotators, both from cool spots and magnetic regions. Contrary to the rotational shear of HD 199178, which is similar in magnitude to that measured on several PMS single fast rotators, the surface differential rotation of HR 1099 is very weak (4 times smaller than solar), though unambiguously detected.

This discrepancy between HR 1099 and HD 199178 is not likely to originate from a difference in the evolutionnary stage of both objects, since they both have left the main sequence and possess a deep convective zone. Another possible origine is the higher mass of HD 199178, and such a difference may indeed partly explain its stronger surface shear, as suggested by other observational studies (Donati et al. 2000, Reiners \& Schmitt 2002). However, this dependence of differential rotation on stellar mass was detected on young dwarfs, and may not hold for more evolved objects with very deep convective zones. The effect that may most likely weaken the surface shear of HR 1099 is the strong tidal forces that it suffers, imposing very efficiently corotation not only to the binary system itself, but also to the rotation within the convective zones of both stars. A possible consequence of these tidal forces is to limit the amount of the surface shear. The theoretical work of Scharlemann $(1981,1982)$ shows that the differential rotation of an evolved component of a RS CVn system is indeed likely to be strongly weakened (though not totally suppressed), in very good agreement with our observations.

The lifetime of photospheric structures (investigated from the comparison of surface images obtained at close-by epochs) is longer on HR 1099 than on HD 199178 (Petit et al. 2003a, 2003b), with most structures of HR 1099 remaining stable over more than 4 weeks, whereas significant changes occur within only 
2 weeks at the surface of HD 199178. This better stability of the photosphere may be related to weaker surface shear of HR 1099.

\section{Future Work}

We are currently carrying out similar studies on a sample of single and binary evolved fast rotators to determine whether the weak surface shear detected on HR 1099 is indeed due to a tidal torque, rather than to other stellar parameters (such as the stellar mass). These new targets include FK Com itself, as well as several RS CVn systems (UX Ari, II Peg and $\sigma$ Gem).

We also plan to pursue the monitoring of both active stars described in the present study with the new generation spectropolarimeters (ESPaDOnS at the CFHT and NARVAL at the TBL) to benefit from increased spectral resolution and $\mathrm{S} / \mathrm{N}$, and to take the opportunity to conduct multi-site studies that provide the most accurate estimates of differential rotation in the case of intermediate rotators. Such high-quality data, along with the long-term monitoring engaged with the present study, should make it possible to test Applegate's (1992) prediction that the intensity of the rotational shear of RS CVn systems may vary during a magnetic cycle, and may be connected with the observed fluctuations of the orbital periods of these objects.

\section{References}

Applegate, J.H. 1992, ApJ 385, 621

Barnes, J.R., Cameron, A.C., James, D.J., Donati, J.-F. 2000, MNRAS 314, 162

Donati, J.-F., Brown, S.F. 1997, A\&A 326, 1135

Donati, J.-F., Semel, M., Carter, B.D., et al., 1997, MNRAS 291, 658

Donati, J.-F., Mengel, M., Carter, B.D., Cameron, A.C., Wichmann, R. 2000, MNRAS 316,699

Donati, J.-F., Cameron, A.C., Semel, M., et al. 2003a, MNRAS (submitted)

Donati, J.-F., Cameron, A.C., Petit, P., 2003b, MNRAS (submitted)

Petit, P., Donati, J.-F., Cameron, A.C., 2002, MNRAS 334, 374

Petit, P., Donati, J.-F., Wade, G.A., et al., 2003a, MNRAS (submitted)

Petit, P., Donati, J.-F., Wade, G.A., et al., 2003b, MNRAS (submitted)

Reiners A., Schmitt J.H.M.M., 2002, A\&A 393, L77

Scharlemann, E.T., 1981, ApJ 246, 292

Scharlemann, E.T., 1982, ApJ 253, 298

Vorontsov, S.V., Christensen-Dalsgaard, J., Schou, J., et al., 2002, Science 296, 101 